\title{
LA REPRODUCCIÓN DE LAS DESIGUALDADES EN LAS TRAYECTORIAS DE MOVILIDAD SOCIAL INTERGENERACIONAL DE LOS JÓVENES MARROQUÍES EN ESPAÑA
}

\section{THE REPRODUCTION OF INEQUALITIES IN \\ THE INTERGENERATIONAL SOCIAL MOBILITY TRAJECTORIES OF MOROCCAN YOUNGSTERS IN SPAIN}

Sofía Laíz Moreira

Resumen: Este artículo presenta los principales resultados de una investigación realizada entre los años 2011 y 2015 y cuyo objetivo fue el de analizar las estrategias de movilidad social intergeneracional de las familias marroquies vinculadas a tres perfiles concretos de jóvenes migrantes: los jóvenes de segundas generaciones, los reagrupados y los jóvenes llegados como menores no acompañados. Todos ellos, inmigrados en la Comunidad Autónoma de Galicia, en España, a principios del presente siglo.

Una metodología fundamentalmente etnográfica y de carácter multi-situado guió un extenso trabajo de campo realizado con diez. familias migrantes cuyos miembros residían en ocasiones dispersos entre Galicia y la provincia de Beni Mellal, en Marruecos. Las mediciones de cambios en las categorías educativas y ocupacionales se realizaron teniendo en cuenta aquellas de los padres y las de los descendientes, es decir, entre generaciones.

* Mesopolhis - Telemme. CNRS, Aix-Marseille Université, SciencePo, France. 
Los resultados mostraron unas trayectorias educativas ascendentes entre las generaciones, pero unos resultados poco alentadores en la inserción laboral de los descendientes, pues éstos reproducían las condiciones de precariedad y fragilidad laboral de los padres, insertados en su mayoría en el sector informal de la economía local.

Palabras claves: jóvenes migrantes; migraciones marroquies; movilidad social intergeneracional; menores no acompañados; $\mathrm{Ga}$ licia; España.

Abstract: This paper presents the main results of a research carried out between 2011 and 2015 which objective was to analyze the intergenerational social mobility strategies of Moroccan families related to young migrants of three main profiles: second generation migrants, reunifies children and and those arrived as unaccompanied minors. All of them settled in the Autonomous Community of Galicia, in Spain, at the beginning of this century.

A mainly ethnographical methodology using a multisided approach has guided a large field work with ten family groups, which members were settled between Galicia and the Province of Beni Mellal, in Morocco. The measurements in educational and professional categories have taken into account the generation of the parents and that of that of children, that's to say, from an intergenerational point of view.

Results have shown upward educational mobility between generations but poor results in regard to offspring's professional insertion, as they reproduced precarious and weak work conditions as their parents, employed mainly in the informal economy.

Key words: young migrants; moroccan migrations; intergenerational social mobility; unaccompanied minors; Galicia; Spain.

\section{INTRODUCCIÓN}

La población marroquí constituye uno de los primeros grupos migrantes en España (López García, 2003; Pumares, 2002). Sus flujos datan de la década de los 70, aumentando su volumen en los 80 y 90, aunque a ritmo menos acelerado (Gómez Crespo, 2004).

La instalación de migrantes en zonas más alejadas de las grandes urbes de desarrollo económico en el mapa estatal obedece generalmente a estrategias particulares de inserción laboral 
(Pumares et al., 2006, 2011). Este es el caso de la Comunidad Autónoma de Galicia, comunidad autónoma limítrofe con Portugal. Es así como los migrantes procedentes de una región en concreto de Marruecos (Beni Mellal) desarrollaron, desde las primeras migraciones iniciadas por los años 80 , una red migratoria en la que el mercado de calle transfronterizo constituiría su nicho de empleo principal.

Los marroquíes en Galicia comienzan a hacerse visibles a finales de la década de los 80 y principios de los 90, según constatan los resultados obtenidos del trabajo de Golías Pérez (2004). Como resalta la misma autora, ésta parece tener especial vinculación con la primera regularización extraordinaria en España del año 1991. Es más tarde, en el año 1995, cuando el crecimiento del número de familias que llegan tras haber residido en otras comunidades como Catalunya, Madrid o Andalucía empieza a tener características de un asentamiento real. Según este mismo estudio la distribución de la población marroquí por provincias responde a una concentración evidente en las provincias de Pontevedra $(49,37 \%)$ y A Coruña $(28,35 \%)$, sobresaliendo para la primera los municipios de Vilaboa, Pontevedra, Vigo y Tui y para la segunda, con una indiscutible concentración en el municipio de Arteixo (Golías Pérez, 2004).

Para este trabajo, y teniendo en cuenta la población de estudio, hemos focalizado la atención en la cohorte que va desde los 15 a los 24 años, considerando la tardía edad de emancipación en España. Para el año 2010, la cohorte de edad seleccionada suponía más del $15 \%$ del total de la población proveniente de este país, con una mayoría de asentamientos también observados en las provincias de Pontevedra y A Coruña ${ }^{1}$.

Este artículo tiene el objetivo de explorar unas migraciones en concreto: los y las jóvenes y sus familias marroquíes procedentes de Beni Mellal inmigrados en Galicia. Dentro de este tema más genérico, nos focalizaremos en los procesos de movilidad social intergeneracional, es decir, en los cambios en las categorías educativas y ocupacionales de padres a hijos. Es por ello que el objeto de estudio se centró en los grupos familiares y no solo en los descendientes, si bien las familias fueron localizadas a partir de la identificación de jóvenes dentro de la franja de edad ya mencionada. Así, si en un primer momento, los jóvenes constituían el objeto

1 Fuente: Instituto nacional de Estadística. 
de estudio inicial de nuestro estudio, más tarde, este análisis nos condujo a un giro más amplio pues en las estrategias individuales se entremezclaron las estrategias de tipo grupal y familiar. Y es que estudiar la movilidad social intergeneracional supone incluir una perspectiva de estudio que va más allá de las trayectorias individuales (Sáiz, 2004).

Nuestra pregunta de investigación se dirigió a contrastar la hipótesis de que las trayectorias de movilidad social de los jóvenes migrantes describen la reproducción de las desigualdades que recaen sobre el colectivo marroquí en España, basándonos en la premisa de que este fenómeno ya ha sido observado en otras comunidades españolas (Pedreno Canovas, 2013; Garcia Borrego, 2011, 2012).

El principal objetivo de este artículo es abordar estos procesos desde el análisis de las trayectorias migratorias familiares, atendiendo a las trayectorias educativas y ocupacionales de varios de sus miembros. Para ello, el análisis se desarrollará desde una perspectiva histórica de cada grupo filial, incluyendo, en ocasiones, a los abuelos de los jóvenes objeto de estudio; si bien, éstos solo se reflejarán en las historias grupales, pues no serán objeto del análisis de los movimientos entre categorías ocupacionales y niveles de estudios según la escala de medición elegida.

\section{MARCO METODOLÓGICO}

Este trabajo se basó en un estudio de tipo cualitativo realizado entre los años 2011 y 2015, aplicando el método de la etnografía multi-situada, en base a entrevistas en profundidad e historias de vida realizadas a diez grupos familiares y realizando el trabajo de campo en dos contextos relacionados a ellas: la comunidad autónoma de Galicia, en España y la provincia de Beni Mellal, en Marruecos. Las familias fueron localizadas a partir de la selección de jóvenes migrantes respondiendo a los tres perfiles de estudio definidos para esta investigación: jóvenes de segunda generación y de generación $1.5^{2}$. Si bien en la investigación inicial se han incluido también los jóvenes llegados a España de forma autónoma, más

2 Definidas por Rumbaut (2004) como las generaciones de hijos de migrantes llegadas al país de inmigración a la edad escolar. 
conocidos como menores no acompañados ${ }^{3}$ o en acogimientos transnacionales ${ }^{4}$, en este artículo, por una cuestión de espacio, nos limitaremos analizar solo los perfiles de jóvenes inmigrados dentro de contextos familiares (generación 1,5) o de segunda generación.

La localización de los informantes fue realizada bajo el efecto de "bola de nieve" a partir del contacto con entidades asociativas. Se ha tenido, para ello, consideración de un equilibro respecto del sexo de los jóvenes contactados en primer lugar, y de la ciudad de instalación de las familias en Galicia, en las localidades de Arteixo, dentro de la provincia de A Coruña, y en la localidades de Tui y Paredes en la provincia de Pontevedra, polos de mayor acogida de flujos migratorios en esta región.

Para completar el abordaje del análisis de las estrategias de movilidad social a partir de la movilidad geográfica, el trabajo de campo en Marruecos fue completado con entrevistas a informantes claves (miembros no-migrantes de la unidad familiar o de la red de parentesco, vecinos, amigos, etc). De esta forma, se buscó la comprensión de la situación de partida que empujó a los actores sociales hacia la movilidad espacial.

Para el presente artículo, el proceso metodológico empleado incluyó la selección de seis historias familiares (de un total de 10 familias) vinculadas a los dos perfiles de jóvenes migrantes detallados más arriba, y con el objetivo de mostrar los procesos de movilidad educativa y laboral entre las generaciones, atendiendo a criterios de edad, de generación y de género, sin por ello prestado atención a una equidad en la selección de los casos según los géneros, pues se ha priorizado el interés de las historias familiares competas, más allá del sexo del joven tomado como sujeto principal de estudio. De esta forma, el hecho de presentar un solo caso masculino dentro de las seis historias presentadas hace referencia a la riqueza de los testimonios en términos de análisis de género y no al sexo de los jóvenes en cuestión. De esta forma, los sujetos centrales de estas narrativas son el o la joven objeto de estudio, pero a través de su historia, accedemos a la historia de los diferentes miembros del grupo familiar, analizando el

3 Los menores no acompañados, se definen según la legislación de extranjería como aquellos que han llegado a la frontera española sin acompañamiento de un tutor legal a cargo.

4 Definidos por Empéz (2015) como los menores no acompañados por sus tutores legales a la migración pero acogidos y guardados por otros miembros de la familia extensa en el contexto de inmigración. 
rol que desempeña su posición en la fratria, las dinámicas jerárquicas en torno a los roles femenino y masculino y acerca del impacto de la edad de llegada de los diferentes miembros de la generación de descendientes sobre sus trayectorias educativas y profesionales.

\section{ESTADO DE LA CUESTIÓN SOBRE ESTUDIOS DE MOVILIDAD SOCIAL EN HIJOS DE INMIGRANTES}

Los trabajos pioneros sobre el estudio de las trayectorias educativas de los hijos de inmigrantes surgen con las investigaciones de Portes y Borocz (1989), Portes y Zhou (1993) y Rumbaut (2004) en Estados Unidos. Uno de los aportes más relevantes de estos trabajos es la clasificación de los hijos de inmigrantes según la edad de llegada al país de inmigración. Dentro de esta misma corriente norteamericana, otro aporte de relevancia radica en las categorías establecidas según la teoría de Valenzuela (1999) sobre los roles de los descendientes en la migración familiar. El autor sitúa a éstos, según su orden etario, en diferentes posiciones respecto de la responsabilidad que sustentan dentro del grupo. Mientras los mayores de la familia representan la figura "subrogada", contribuyendo al ingreso familiar y con poder de decisión sobre la vida de los hermanos más jóvenes, los hermanos intermedios ejecutan el rol de "tutores" o "mediadores" en tanto ejercen de nexo entre el sistema escolar y los padres. Esta categoría es aplicable a familias con lengua materna diferente de la de la sociedad de instalación, si bien los diferentes roles entre descendientes no se explican únicamente en relación al manejo del idioma.

Algunos estudios en Europa ya han comparado las trayectorias de movilidad social de los inmigrantes de primera y segunda generación en relación a aquellas de los autóctonos (Meurs, Simon et Pailhé, 2006; Portes et al., 2009; Miguélez et al., 2011). Los resultados muestran que los hijos de inmigrantes se enfrentan aun hoy en día a serias dificultades en los procesos de inserción socio-laboral al mercado de trabajo. En términos generales, los indicios de movilidad social hablan de una movilidad que se presenta más en términos ocupacionales que reales, ya que se trata de generaciones con un mayor nivel educativo y de escolarización, pero que reproducen el estatus social de sus padres a pesar del incremento general en las habilidades sociales y ocupacionales. 
En España, los trabajos de Pedreño Cánovas (2013) en Murcia y García Borrego (2012) en Madrid han analizado en profundidad la problemática de la reproducción de las desigualdades sociales de padres a hijos en el caso de las familias inmigrantes. En ambos estudios queda constatado como los procesos de segregación social que recaen sobre las poblaciones inmigrantes en España tienen como consecuencia un impacto decisivo en las trayectorias educativas de los hijos de inmigrantes y más tarde, en las posibilidades de producir una movilidad social intergeneracional ascendente, reproduciendo inevitablemente las condiciones de precariedad laboral y, en la mayoría de los casos, apenas incrementando o incluso decreciendo los niveles de estudios. En los últimos años el trabajo de Bayona-iCarrasco et al. (2020) ha aportado luz al estudio de las trayectorias escolares de hijos de inmigrantes desde un estudio realizado en Catalunya, así como los estudios de Gil-Hernández y Gracía (2018), González-Ferrer y Cebolla-Boado (2018) y Miguel y Solana (2017) a nivel estatal.

Fuera de España y vinculados a al estudio de las trayectorias intergeneracionales de movilidad social en concreto, existen actualmente trabajos muy recientes que han logrado avanzar en el estudio de estos procesos vinculados a las migraciones internacionales, entre los que resalta el reciente trabajo de Oso Casas et al. (2018) en Buenos Aires.

Pero sin duda, las investigaciones de Aparicio y Portes (Portes et al., 2011; Aparicio y Portes, 2014) han conformado el más extenso dentro del estudio de las segundas generaciones en España y se centra en las comunidades de Madrid y Catalunya. Las conclusiones más importantes hablan de una movilidad social inter-generacional diferenciada según origen étnico. Tomando como referencia las teorías sobre la asimilación de las segundas generaciones en los Estados Unidos, particularmente el trabajo de Portes y Zhou (1993), los resultados destacan como la diferente función de los factores condicionantes en tales trayectorias (capital social, estructura familiar, discriminación, asimilación cultural e identidad) configuran las diferentes estrategias y necesariamente explican el mayor o menor éxito en el acceso a una posición social favorecida o segmentada. En resumen, en los casos en los que el capital cultural es menor, la movilidad social es posible pues el grado de impacto de las dinámicas de segregación social que recaen sobre el colectivo migrante combinadas con las condiciones impuestas por la política 
educativa en España permiten la mejora significativa del nivel de estudios de padres a hijos. Por el contrario, en el caso de los padres migrantes con nivel de estudios más elevado, los hijos no logran vencer los diferentes obstáculos que impone la etno-estratificación social en España, disminuyendo el nivel de estudios y ocupando categorías socio profesional inferiores a las alcanzadas por los padres en el país de origen.

Asimismo, cabe mencionar el enfoque de estudio de Labrador, Blanco y Ortiz (2007) que analizan los procesos de discriminación en las trayectorias de incorporación a la vida adulta de los jóvenes hijos de inmigrantes. Entre las conclusiones más importantes encontramos aquella que habla de dos tipos de salidas identitarias de los hijos de inmigrantes, siendo la más recurrente la "huida expresiva", donde el joven, frente al rechazo de la sociedad de acogida, se posiciona en el otro extremo, haciendo eco de todos los prejuicios negativos que recaen sobre él, como forma de identificación social (Labrador et al., 2007, p.181).

No debemos olvidar mencionar el trabajo de García Castaño y Rubio (2013), quienes sacan a la luz cómo la lógica del fenómeno de concentración de alumnado extranjero en ciertos centros escolares y las políticas de atención a la diversidad que en ellos se aplican, contribuyen a una construcción de la diferencia, una segregación escolar, que explica en gran medida las desigualdades en términos educativos entre autóctonos y alumnado inmigrante.

\section{DEFINICIÓN DE CONCEPTOS CLAVES PARA EL ESTUDIO DE LA MOVILIDAD SOCIAL}

Antes de pasar a analizar los casos presentados para este estudio, es necesario recordar algunas definiciones básicas para comprender nuestra perspectiva dentro del análisis de la movilidad social. Para comenzar, entendemos la movilidad laboral como los cambios individuales de posiciones en una escala de categorías laborales, o bien a movimientos entre sectores de actividad económica o geográfica (Miguélez et al., 2011, p. 135), es decir, al ascenso, descenso o reproducción que se produce entre diferentes categorías ocupacionales (Echeverría, 1999). La movilidad educativa, por su parte, reflejaría el cambio entre categorías equivalentes a los diferentes niveles 
de cualificación, y, desde el análisis cuantitativo, nos indicaría el grado de correlación entre el nivel de escolarización de los padres y de los hijos (Sánchez Hugalde, 2003, p. 3). Precisamos, más aún, explicar cómo entendemos los diferentes movimientos entre categorías para medir el tipo de movilidad educativa y/o ocupacional. De esta forma:

- la movilidad educativa, se entendería como el proceso por el cual el sujeto de estudio muestra una mejora o una desmejora en el nivel formativo respecto de aquel que ostentan sus padres.

- la movilidad ocupacional, cuando el sujeto de estudio obtiene una categoría ocupacional mejorada o en contraste, desmejorada, en comparación a la de sus padres.

- Por último, la reproducción del nivel educativo, cuando el nivel formativo se reproduce de padres a hijos y reproducción del nivel ocupacional cuando la categoría ocupacional es la misma entre ambas generaciones.

Cabe señalar que la escala seleccionada para realizar la medición de los cambios entre generaciones corresponde a la Clasificación Socioeconómica Europea (ESeC) .

Una vez revisados los conceptos teóricos y la terminología a utilizar a lo largo de nuestro análisis, pasaremos, en el apartado a continuación, a presentar las trayectorias educativas y ocupacionales de las diferentes generaciones de cada grupo familiar, tomando siempre al joven de origen migrante como objeto central de estudio.

\section{TRAYECTORIAS DE MOVILIDAD SOCIAL INTERGENERACIONAL A PARTIR DE LAS HISTORIAS FAMILIARES}

El presente apartado presenta los resultados del trabajo etnográfico realizado con diez familias migrantes. Por una cuestión de extensión, hemos escogido seis de ellas para reflejar las trayectorias detalladas

5 El sistema ESeC fue teorizado por Goldthorpe, desde la corriente neoweberiana, y presenta una categorización que agrupa aquellas ocupaciones codificadas con tres dígitos de la CIUO-88, logrando mantener las categorías en una posición similar dentro del mercado de trabajo y, al mismo tiempo, permitiendo otras agrupaciones más sintéticas que reducen la complejidad de la clasificación (Informe España CECS, 2011: 309). 
de los casos más representativos. Para ello, un procedimiento de anonimización ha asignado pseudónimos tanto a los y las jóvenes como a los diferentes miembros de sus familias citados en cada historia familiar.

Comenzaremos con la historia familiar de Baia. Se trata de una joven originaria de un poblado de la provincia de Beni Mellal en Marruecos. Ésta describe una movilidad educativa ascendente respecto de los niveles escolares alcanzados por sus padres. Su situación ocupacional, si bien es precaria, supera la condición de su madre (inactiva) y de su padre (vendedor ambulante) pues la joven ha dado el paso al mercado primario. Veamos cómo se produce este proceso dentro de la historia familiar:

\section{Historia familiar 1: Baia}

Baia es la hija mayor de una familia compuesta por el matrimonio y dos hermanas. Su llegada a Galicia tuvo lugar en el año 2005, cuando la joven tenía 15 años. Su padre, Ahmed, llevaba residiendo en la localidad de Tui por casi una década, después de haber vivido en Italia, junto a sus hermanos emigrados allí y en Alicante, donde trabajó temporalmente en la recogida de uvas. Ahmed comenzó su periplo por Turín en el año 1993 atraído por el éxito que habían conseguido sus hermanos mayores. Allí Ahmed trabajaba en el sector de la piedra, donde había logrado insertarse por sus redes familiares también empleadas dentro de este sector. Alrededor del año 1996 llegó a Galicia donde se empleó en el mercado ambulante, recorriendo varias localidades gallegas (Tui, Ferrol, Pontevedra, A Coruña, Santiago, Vigo) con la mercancía que traía de Portugal. Su llegada estuvo guiada por la ruta que había seguido el marido de su prima, así como otros vecinos de su pueblo de origen, entre ellos, el primero en llegar a la Comunidad gallega que residía en la ciudad de Pontevedra desde hacía más de 35 años.

La reagrupación familiar no pudo realizarse hasta el tercer intento, siendo denegada en dos ocasiones. En ello se demoró 9 años, hasta que en 2005 pudo traer a su mujer e hijas. Baia y su familia son originarios de la aldea de Ouled Youssef, un poblado rural cercano a la ciudad de Beni Mellal, en la región de Tadla Azilal. Amhed apenas había llegado a cursar algunos años de la educación primaria, y Soumia, su mujer, solo estuvo escolarizada tres años. La tardía reagrupación de Baia hizo que su llegada al sistema escolar gallego fuera extremadamente difícil, especialmente debido al desconocimiento absoluto de las lenguas 
oficiales habladas en Galicia y a la diferencia de conocimientos de base. Con todo, Baia contaba con la ayuda de otras niñas llegadas de poblados próximos a su aldea natal que ejercían el rol de traductoras e intermediarias con el profesorado para favorecer su integración en el centro escolar. Baia llegó a cursar los años de enseñanza secundaria obligatoria con muchas dificultades para aprobar los diferentes niveles. Ello hizo en gran medida que su trayectoria educativa se terminara incluso antes de culminar el ciclo de la E.S.O, dejando sin aprobar la última asignatura cuya fecha de examen perdió por viajar a Marruecos junto a su familia. La joven, por tanto, no cuenta siquiera con el diploma de graduado en educación secundaria. Su carencia de titulaciones impuso probablemente el mayor de los obstáculos para conseguir insertarse en el mercado de trabajo local y su trayectoria ocupacional se encaminó hacia el sector del empleo doméstico, guiada por las redes sociales comunitarias y familiares cuyas mujeres estaban mayoritariamente empleadas en este sector. Así fue como, con 17 años, Baia comenzó a trabajar como empleada doméstica en una vivienda de la ciudad de Vigo, sin ser regularizada su situación laboral, es decir, dentro del mercado informal del empleo doméstico. Ello supuso su cambio de residencia a la vivienda de uno de sus tíos que residía en esta ciudad, regentando una de las dos carnicerías con productos "halal" ${ }^{6}$ de la ciudad. Allí ha continuado trabajando desde hace cinco años. Su interés por el estudio no ha vuelto florecer, a excepción de la preparación del examen de conducir, una de las mayores metas para su padre y para la joven pues este permitiría facilitar la movilidad de la familia. La trayectoria educativa de su hermana, sin embargo, parece presentar menos dificultades que la de Baia, quien al llegar a una edad más joven no tuvo que enfrentarse a tantas dificultades en la adaptación a la escuela y planea realizar un ciclo de formación profesional en enfermería.

En la historia de Baia vemos cómo los obstáculos impuestos por la política migratoria y de integración de los inmigrantes en España, como bien ha señalado Gil Araujo (2007), pueden causar un retraso considerable en la llegada de los descendientes reagrupados y con

6 "El término halal (también transliterado al l o halaal) hace referencia al conjunto de prácticas permitidas por la religión musulmana. Aunque el término en sí engloba a todo tipo de prácticas, es comúnmente asociado a los alimentos aceptables según la ley islámica" (Wikipedia, disponible en: http://es.wikipedia. org/wiki/Halal). 
ello, el abandono de la trayectoria educativa de forma prematura. Se produce, no obstante, una movilidad educativa y laboral ascendente, si bien en condiciones de extrema precariedad pues, como también han constatado otros estudios en España (Portes et al., 2011, Aparicio y Portes, 2014), en poblaciones migrantes con bajo nivel educativo, una mejora del nivel de estudios resulta posible con el nivel mínimo requerido a partir de la obtención del diploma de educación secundaria obligatoria. En el caso de Baia, su nivel educativo es mayor que el de sus padres y la obtención de un contrato de trabajo puede considerarse una mejora en las condiciones laborales, si bien no ha siquiera obtenido el título de enseñanza secundaria obligatoria. Esta se configura como una de las principales razones que conducen a la joven hacia los puestos más bajos de la escala social, bloqueando cualquier posibilidad de formación futura.

En segundo lugar vemos en la trayectoria educativa de Baia el rol que juega la influencia de la familia como motor principal en la motivación hacia los estudios y da cuenta del lugar que ocupa el valor de la educación para los padres y su impacto en la trayectoria de los hijos. En efecto, la idea de una transmisión del capital cultural en forma de "disposición hacia la educación" es una de las principales premisas de la teoría de la reproducción social de Pierre Bourdieu (Bourdieu y Passeron, 1970), también observado en trabajos en España como los de Pedreño Canovas (2013) y García Borrego (2012). En su caso, la falta de incentivos familiares para que la joven culminara sus estudios, aparejado a la necesidad económica, han favorecido al abandono de la trayectoria educativa.

Un segundo patrón de movilidad educativa y ocupacional representa uno de los casos más optimistas, el caso de Ahlem, el cual refleja una movilidad educativa ascendente y una movilidad ocupacional en la misma dirección, produciéndose un verdadero salto ocupacional entre generaciones, pues Ahlem obtiene una titulación de educación superior en el nivel de máster universitario.

Historia familiar 2: Ahlem

Ahlem es originaria del poblado de SoukSebt. Su padre, quien cuenta con un nivel de estudios primarios, se encuentra dentro del grupo de los primeros migrantes marroquíes llegados a Galicia. Éste se había instalado en la localidad de Cangas donde trabajaba como vendedor ambulante de alfombras y otros enceres varios que traía desde Portugal. Su mujer e hijas fueron reagrupadas años más tarde. 
La llegada de Ahlem y sus hermanos a la escuela de Cangas no pasó desapercibida. Eran los primeros niños de origen marroquí en toda la parroquia y el trabajo de su padre hacía que toda la comunidad local los conociera como "los hijos del vendedor de alfombras". Allí se integraron de forma paulatina y con un gran apoyo del profesorado, asegura Ahlem. Si bien las dificultades del idioma no fueron menores, la adaptación a la escuela primaria en Cangas ha dejado un grato recuerdo en la memoria de Ahlem. Sin embargo, fue el cambio de centro lo que parece haber claramente influido en las trayectorias educativas de la joven y de sus hermanos. Tras la detección de una grave enfermedad en su madre, la familia decide trasladarse a la localidad de Vigo, más próxima al centro hospitalario. El nuevo centro escolar ubicado en un contexto más urbano no ofrecía ya el apoyo que habían gozado en la pequeña escuela de Cangas y el testimonio de Ahlem denota escenas de discriminación y de aislamiento experimentadas por algunos de sus hermanos. Esta situación parece haber influido especialmente en la trayectoria educativa de la hermana mayor de la fratría, quien decidió dejar los estudios. Ahlem y dos de sus hermanos, sin embargo, continuaron estudiando. Cuenta la joven que si bien los resultados escolares le permitían acceder al bachillerato sin mayor inconveniente, la orientación que recibía del centro escolar la guiaba hacia la formación de tipo profesional. Ahlem recuerda como la insistencia de su hermana mayor le había ayudado a seguir adelante respectando sus deseos. Es por ello que la joven decidió seguir la vía de los estudios superiores, como lo había soñado. Ahlem pudo culminar con su bachillerato y escogió la diplomatura de trabajo social. Al acabar estos estudios, intentó encontrar un puesto adaptado a sus expectativas, pero dada la necesidad económica y la falta de empleo joven en Galicia la joven decidió buscar otras vías de inserción y consiguió un contrato de trabajo como comercial de calle en una empresa de telefonía móvil. Mientras trabajaba en esta empresa ubicada en Vigo, Ahlem decidió proseguir con una especialización para dar más orientación a su carrera profesional y se decantó por los estudios migratorios, realizando un Master en la ciudad de A Coruña. Al culminar el Máster, Ahlem intentó nuevamente orientar su trayectoria ocupacional hacia su ámbito de formación sin éxito, razón por la cual la joven continuó trabajando como comercial de calle. Las expectativas educativas del padre de Ahlem fueron cumplidas con creces por su hija, si bien la situación del mercado de trabajo local y posiblemente cierta dosis de etno-estratificación no pudieron evitar que su trayectoria se orientara hacia un puesto de menor cualificación que el adecuado a su nivel 
de estudios. Una de sus hermanas mayores no había podido ingresar al sector de la sanidad pública ni privada a causa de portar el velo. Ésta se insertó rápidamente en el sector de la hostelería, si bien cuenta con un diploma de formación profesional en enfermería. Ahlem asegura que sus posibilidades fueron claramente diferentes a la de sus primas en origen y ello se debe al cambio de percepción de su padre sobre el empleo femenino. Es por ello que la joven otorga a la migración un lugar fundamental en las oportunidades educativas y profesionales a las que ha podido acceder en comparación con sus pares no migrantes.

Ahlem ilustra el espíritu de superación que se observa en la actitud de otras jóvenes mujeres marroquíes observadas en este estudio. Esta actitud, en el caso de Ahlem parece ser el reflejo del ejemplo de superación mostrado por su hermana mayor, heredera de las obligaciones propias del rol materno tras el fallecimiento de su madre. Este fenómeno, ya estudiado como vimos por Valenzuela (1999), refiere a un rol tutor que empuja a los hermanos y hermanas menores a personificar otras obligaciones dentro del grupo, tales como en la responsabilidad de representar la promesa a la movilidad social, como es el caso de la joven protagonista de esta historia. Como muestra la historia familiar, existe un evidente impacto de lo que otros estudios han constatado como factores determinantes en las trayectorias educativas de los hijos, como son la edad de llegada el nuevo sistema escolar y la posición ocupada en la fratria (Portes et al., 2011). La historia de Ahlem nos revela además elementos que tienen que ver con el género de los descendientes, la discriminación racial y el estigma en torno al hecho de ser musulmán.

Sobre el primero de estos puntos, vemos como los hermanos de Ahlem, como muchos otros jóvenes marroquíes, abandonaron la senda educativa para insertarse en el mercado de trabajo tras la enseñanza obligatoria. El hecho de que los hijos varones muestren un desempeño menos aplicado a los estudios es un tema que se presenta recurrente en los resultados de investigaciones sobre trayectorias educativas (Boukoubza, 2005), indiferentemente del origen nacional o inmigrante (García Castaño, 2012). Éstos suelen optar por la vía de entrada más rápida al mercado de trabajo, impulsados por la presión social que les obliga a responder a obligaciones dentro del rol productor familiar.

Cuando intentamos explicar el fenómeno de la discriminación hacia la población marroquí en España, no podemos olvidar lo 
que Rodríguez-Rech y Rodríguez-García (2020) nos recuerdan en su trabajo sobre el estigma de la musulmaneidad, pues en el ejemplo de Ahlem quedan sentadas las bases sobre el mecanismo que delimita "los marcadores visibles y estereotipados que sitúan inmediatamente a la persona que es o parece musulmana en la posición del "otro", dando pie al trato diferencial" (Ibid, p. 1). El uso del velo en el caso de la joven pone en evidencia como los símbolos culturales son tomados como objetos legitimadores de tal otredad, contribuyendo a la creación y el mantenimiento de distintas formas de poder (Mateo Dieste, 2018).

Vemos también como la discriminación de género se ve en este ejemplo doblemente representada, pues toma la categoría étnica subordinada para beneficio de una categorización social doblemente discriminante (Ahlem en tanto que mujer trabajadora y en tanto musulmana) excluyendo a la joven de este espacio social por el hecho de ser musulmana pero también y particularmente, por el hecho de ser mujer-musulmana.

En el próximo ejemplo, analizaremos un segundo modelo de movilidad educativa observado en nuestra población de estudio: aquel que refleja la reproducción del nivel formativo de padres a hijos. Entre los casos que se corresponden con este patrón, ubicamos aquellos que, al momento de la entrevista, se encontraban igualando el nivel educativo de sus padres, pese a que en ambos ejemplos las trayectorias educativas no estaban terminadas. El caso de Chadia es uno de ellos. No obstante, es importante remarcar que su estrategia educativa preveía una movilidad educativa de tipo ascendente superando el nivel de educación secundaria con la continuación de estudios superiores:

\section{Historia familiar 3: Chadia}

Chadia nació en Vigo, en la provincia gallega de Pontevedra. Su padre, Hicham, llegó a Galicia alrededor del año 1986. Había ya pasado por varios países anteriormente, entre ellos Arabia Suadi, Irak, Ucrania y Francia. Hicham es un hombre con estudios secundarios terminados y ha proseguido la orientación de los estudios religiosos. Es un hombre muy respetado en la comunidad marroquí de Redondela y alrededores, donde la familia reside desde hace varios años. Hicham ha llegado a esta pequeña localidad pontevedresa para ocupar el puesto de Imán, como cabeza de la institución islámica local. Hace tan solo unos años, ha accedido a insertarse en una empresa de limpieza municipal 
produciéndose una movilidad ocupacional desde el mercado secundario al primario.

Chadia, al momento de la entrevista, contaba con 19 años. Su relato refleja el sueño de un futuro lleno de oportunidades. La madre de Chadia, Hjadija, no ha podido estudiar, siendo originaria de una pequeña aldea de Beni Mellal donde la lejanía respecto del centro escolar imposibilitó que siguiera una trayectoria educativa más allá de los primeros años de la educación primaria. El hecho de no haber tenido la oportunidad de estudiar ha hecho que la educación sea para Hjadija una meta fundamental en la educación de sus hijas. Chadia no ha sufrido el impacto de una adaptación entre sistemas escolares pues ha nacido en Galicia. Su buen desempeño escolar, muy vigilado desde el ámbito familiar, ha sido una buena razón de continuar con los estudios secundarios, encontrándose actualmente cursando el bachillerato. Chadia aspira a una carrera universitaria de alto nivel. En ello influyen y mucho las ilusiones que sus padres ponen en su trayectoria educativa y que orientan sus deseos hacia la culminación de los estudios en el extranjero, concretamente en Arabia Saudita. Al momento de la entrevista, Chadia proyectaba sus ilusiones de carrera en proseguir con la licenciatura de arquitectura y desplazarse a este país, pese a que las condiciones socio-económicas familiares no parecían poder afrontar una inversión de este tipo.

El ejemplo de Chadia, ilustra de manera clara la influencia del capital humano familiar y de las expectativas de los padres en las trayectorias educativas de los descendientes, entrando en discusión con otros trabajados que han revelado la importantísima influencia de las expectativas familiares en las trayectorias seguidas por los hijos (Bourdieu y Passeron, 1970; Pedreño, 2007, 2010). La trayectoria de Chadia refleja, asimismo, el desajuste que puede darse entre las expectativas de los jóvenes y las posibilidades reales de consolidar sus sueños educativos teniendo en cuenta los recursos económicos con los que cuentan y cuyas barreras se consolidan en la posición segmentada que pudieron adquirir los padres. Este hecho, como ya concluyó Pedreño, entre otros autores, revela los indudables límites en el apoyo que éstos pudieron prestar a las trayectorias educativas de sus hijos.

Una segunda historia familiar que ilustra el patrón de la reproducción del nivel educativo inter-generacional se revela en la trayectoria de Fatiha y de su familia: 
Historia familiar 4: Fatiha

Fatiha es una joven de segunda generación marroquí, nacida en la localidad gallega de Tui, donde su padre decidió asentarse en el año 1993 siguiendo los consejos de su hermano, instalado en Galicia años antes, uno de los precursores de uno de los nichos de empleo marroquí en esta comunidad: el mercado ambulante y transnacional entre Portugal y Galicia. Mohamed realizaba estudios de bachillerato en Marruecos cuando su hermano le convenció de las ventajas de la migración a España. El joven, en aquella época con tan solo 19 años, abandonó sus planes de carrera universitaria en Marruecos para migrar a Galicia e incorporarse en la actividad del comercio ambulante. El cambio de estructura social sitúo a Mohamed en una posición muy rebajada dentro de la escala social de ambos países, pues su actividad ocupacional en Galicia no dejaba de representar un sector informal, del mercado secundario local y altamente segmentado. Ello ha llevado a Mohamed a arrepentirse de haber dejado los estudios, sobre todo frente a la situación del mercado trabajo local hacia finales de la década del 2000y las dificultades que afronta la familia con la crisis económica en Galicia. Con todo, Mohamed ha logrado un status social reconocido dentro de la población marroquí residente en Tui pues es el líder de la comunidad islámica local donde desempeña un papel social importante. Mounia, su mujer, no tuvo la oportunidad de estudiar más allá de algunos cursos de la educación primaria pues tuvo que abandonar su trayectoria formativa tras la migración de su padre a Italia y la enfermedad de su madre que requería de su cuidado y de apoyo en las actividades domésticas. Si bien sus hermanos y hermanas pudieron realizar estudios superiores, ella fue la elegida para ocuparse de su madre enferma. Su frustración en este ámbito ha contribuido a crear una transferencia de expectativas educativas en las trayectorias de sus hijos. Mounia se dedica al cuidado de los hijos y a la actividad doméstica, si bien, de forma intermitente a remplazado a una joven de su comunidad residente en la localidad de Tui que trabaja como empleada doméstica. Ambos padres depositan, por todo ello, grandes esperanzas de movilidad educativa en sus descendientes, especialmente en Fatiha, primogénita y exponente más próxima de una posible movilidad social a partir de la educación.

Fatiha, tenía 15 años al momento de la entrevista y se encontraba cursando el ciclo de E.S.O. Los recuerdos de la tierra de la que es originaria su familia forman parte de un importante patrimonio familiar. La pertenencia de Fatiha al país de nacimiento de 
sus padres es constantemente alimentada con anécdotas y viajes estivales a partir de los cuales la joven y sus hermanos menores han aprendido a conocer sus raíces. Fatiha tiene planes de carrera universitaria, de cumplir el sueño al que tuvo que renunciar su padre. Sus planes son de continuar con el bachillerato y más tarde acceder a los estudios de arquitectura, pero la situación económica familiar parece presagiar un cambio de rumbo inminente. La familia se plantea la posibilidad de retornar a Marruecos, pues la situación laboral de Hicham es lo suficientemente delicada en el mercado ambulante local para pensar en un cambio de escenario. La crisis económica en Galicia ha añadido muchas dificultades a la estrategia familiar de supervivencia. La falta de trabajo producida por la bajada de ventas cuestiona la idea de volver a Marruecos, donde las condiciones estructurales parecen haber mejorado notablemente desde la partida de Mohamed a España, donde la familia cuenta con una vivienda y redes sociales de mayor poder en el acceso al mercado de trabajo local y donde el coste de la vida es mucho menor.

El caso de Fatiha, al igual que el de Chadia, da cuenta de la influencia de los dos factores citados: las expectativas familiares de movilidad inter-generacional por un lado, y la fragilidad de una población migrante que ocupa los peldaños más expuestos a los vaivenes económicos, como segundo factor determinante. La situación de precariedad en el empleo de las primeras generaciones, como vemos nuevamente, puede presentarse como principal obstáculo para dar el apoyo necesario a los planes de estudios proyectados. De esta forma, y en dialogo con los trabajos ya mencionados, esta experiencia nos vuelve a confirmar que la reproducción de las desigualdades parecen tener una íntima relación con la posición que ocupan los padres en la sociedad de acogida, posiciones que ya sabemos social y étnicamente estratificadas, y que se presentan como los mayores frenos a los deseos de movilidad en los hijos, pues estos tienen que escoger la vía de la salida precoz del sistema educativo para aportar financieramente al grupo familiar.

En la historia familiar narrada vemos claramente este proceso representado en el hecho de que si bien Hicham, padre de Fatiha, ha llegado a Galicia con un nivel de estudios secundarios, su inserción inmediata en el nicho étnico local ha condicionado su acceso a redes sociales útiles, que le permitieran escapar de una estructura laboral etno-estratificada. Sumado a ello, la situación de crisis del empleo de finales de la década 2000, hizo surgir la idea de un cambio de 
rumbo en el proyecto migratorio familiar hacia el país de origen, lo que podría generar un impacto decisivo sobre la continuidad de la trayectoria educativa de su hija. Pese a ello, los resultados de esta trayectoria no pueden evaluarse en base a la etapa escolar en la que se encontraba Fatiha al momento de la entrevista.

El quinto caso, el de Malika, nos vuelve a conducir hacia el modelo mayoritario observado entre nuestra muestra de estudio, el de una movilidad educativa ascendente, pero nos aporta elementos para comprender las estrategia que los jóvenes y sus familias ponen en marcha para evadir las citadas barreras a la movilidad social impuestas por los procesos de estratificación étnica. Malika ha culminado el bachillerato y se propone continuar con estudios de formación profesional:

\section{Historia familiar 5: Malika}

Malika es una joven de origen marroquí. Su llegada a la localidad de Arteixo, donde actualmente reside junto a su madre, uno de sus hermanos mayores y su hermano menor, se produjo en el año 2003. Su familia es natural del poblado de origen bereber de Tagzirt, en la provincia de Beni Mellal, a los pies del Atlas occidental. Su padre llegó a Arteixo para cubrir el puesto de Imán, como cabeza de la institución religiosa musulmana en la zona y alrededores. La reagrupación familiar, sin embargo, se produjo demasiado tarde para poder permitir que los hermanos de Malika — ya mayores de edad- pudieran también migrar a Galicia.

El padre de Malika tenía un nivel de estudios secundarios según el modelo de enseñanza original. Su madre apenas había cursado algunos años de la escuela primaria. Los hermanos mayores de Malikase encontraban ya trabajando en Marruecos cuando el padre de Malika reunió los requisitos para efectuar la reagrupación familiar. Su hermano mayor obtuvo el título de filología inglesa y trabaja como empleado de la policía estatal en la ciudad de Rabat, en la sección de extranjería. Su otro hermano residente en Marruecos, terminó la educación secundaria y realizó una formación profesional en gastronomía. Trabaja como cocinero en un conocido establecimiento de gastronomía de la ciudad de Beni Mellal. El hermano mayor de Malika que reside en Galicia, sin embargo, no pudo proseguir su trayectoria educativa más allá de la E.S.O, pues llegando en edad adolescente encontró terribles dificultades para continuar con los estudios más allá de la educación obligatoria. Actualmente trabaja como operario de cadena en una fábrica de alimentación en la ciudad de Arteixo. 
Malika, sin embargo, tiene expectativas de convertirse en educadora infantil y luego en psicóloga. Al llegar con tan solo ocho años, su integración en la escuela no fue tan traumática como la de su hermano mayor. Las dificultades que encontró se vincularon principalmente al aprendizaje del idioma. A pesar de ello, la joven ha podido obtener su diploma de graduado en educación secundaria sin mayores inconvenientes y con muy buenos resultados escolares. Actualmente se encuentra realizando las pruebas de acceso a un ciclo de grado medio en educación infantil, pero su estrategia es de acceder, con este primer diploma, a un ciclo superior que le dará acceso a la universidad. Malika ha optado por evitar las pruebas de selectividad y buscar la vía más segura para luego poder entrar a la carrera de psicología, lo cual le permitiría, mientras tanto, ir obteniendo titulaciones válidas para comenzar a trabajar.

La historia de Malika resulta de gran interés pues refleja una estrategia educativa dirigida a sortear las dificultades económicas familiares y la carencia de apoyo financiero. Podría tratarse por ello de un caso de salto generacional por la gran voluntad de superación de esta joven, que no se da por vencida pese a las barreras impuestas por el marco estructural. Malika se plantea acceder a estudios universitarios mediante el acceso a titulaciones intermedias que le permitan obtener su propia fuente de ingresos para poder combinar estudios con trabajo. La historia de esta joven y sus hermanos también vuelve a recordarnos el impacto decisivo de la edad de llegada al nuevo sistema educativo, es decir, del momento, dentro de la trayectoria educativa, en el que se produce la migración, siguiendo los análisis de Rumbaut (2004).

Pasando a un nuevo patrón de movilidad encontramos la movilidad educativa ascendente junto con la reproducción de la categoría ocupacional alcanzada por los padres. Este modelo refleja un proceso de estancamiento ocupacional pues el retorno de los hijos a la actividad tradicional de los padres (el sector del comercio ambulante). Es importante resaltar que estos casos se identifican en su totalidad con sujetos varones. La última de nuestras historias describe este ejemplo y corresponde al caso de Simo:

\section{Historia familiar 6: Simo}

Simo llegó a Galicia, a la localidad de Tui en el año 2004, cuando tenía 12 años, junto a su madre y sus hermanos meses más tarde que su padre consiguiera los papeles para la reagrupación 
familiar, tras obtener su residencia y autorización de trabajo con la regularización del año 2000. El padre de Simo había vivido anteriormente en Italia durante cinco años y residía ya en Galicia desde hacía cuatro siguiendo el rastro del primo de su mujer, vendedor ambulante en Portugal y Galicia, asentado en Tui desde hacía varias décadas. El padre de Simo era agricultor en Ouled Youssef, su localidad de origen; vendía verduras y frutas en el mercado local. Simo, sus padres y sus hermanos vivían en la vivienda de sus abuelos. Al llegar a Tui, el padre de Simo se empleó, igual que el primo de su mujer, en el mercado ambulante. El padre de Simo no tenía prácticamente estudios, apenas había comenzado con la educación primaria y no la había terminado. La madre del joven era prácticamente analfabeta.

La migración se produce, según sus palabras, para mejorar las oportunidades de futuro de los hijos. El proyecto migratorio y de movilidad social de los padres de Simo es de retornar a Ouled Youssef cuando sus hijos hayan conseguido un puesto de trabajo en España, lo que les permitiría obtener mejores ingresos familiares a partir del envío de remesas a origen. Es por ello que la migración de la familia se planteó cuando aún los hijos estaban en edad escolar, para posibilitar una mejor inserción al mercado local.

La trayectoria educativa de Simo encontró algunos obstáculos en un primer momento, dadas las dificultades del idioma y las diferencias en los conocimientos de base, lo que hizo que el joven repitiera el curso en dos ocasiones. Con todo, Simo ha logrado terminar la enseñanza secundaria obligatoria y actualmente se dedica a estudiar español, a la espera de que le llamen para realizar un curso dentro del Programa de Cualificación Profesional Inicial (PCPI) en la rama de electrónica o mecánica, pues quisiera obtener un diploma de formación profesional para poder acceder a un puesto de trabajo. Mientras tanto, Simo trabaja algunos días en el mercadillo de Portugal, junto a su padre y su hermano. Si bien sus padres insisten en que acabe los estudios en Galicia, sus planes individuales barajan la posibilidad de regresar a Marruecos para realizar una formación de mecánica. Simo no sabe bien qué hacer, pero frente a la falta de plazas en la formación solicitada y la falta de posibilidades en el mercado local, suele fantasear con la idea de un retorno a su pueblo natal.

El caso de Simo ilustra con claridad la diferencia de expectativas educativas y profesionales que se plantean desde el prisma del análisis de género, ya desarrollado por autores en otros países, como en el análisis desarrollado por Boukhobza (2004) que pone 
de manifiesto el mejor desempeño de las niñas y su relación con el rol femenino en las familias magrebíes relacionado con el ámbito doméstico. Vemos como los roles establecidos de lo masculino y lo femenino en la familia de este joven le conducen a priorizar la salida del sistema educativo hacia el mundo laboral (la esfera de lo público y exterior) en contraposición a la esfera de lo domestico y privado. Asimismo, refleja cómo estas trayectorias educativas truncadas son el principal motivo de un estancamiento en el proceso de movilidad social y la reproducción de la categoría profesional de padres a hijos dentro del mercado informal gallego.

\section{CONCLUSIONES}

Cuando analizamos las historias presentadas encontramos unas estrategias marcadas temporalmente por la política de reagrupación familiar en España. Dichas limitaciones dejan sentado su efecto sobre los grandes retrasos impuestos a los proyectos migratorios familiares, con un impacto innegable en la edad de llegada de los descendientes al nuevo sistema educativo (Gil Araujo, 2007).

De esta forma, las diferencias acerca del impacto del proceso migratorio familiar parecen ser evidentes si comparamos el caso de los jóvenes llegados con sus familias en edad escolar y los jóvenes de segunda generación nacidos en España. Estas diferencias apuntan a la decisiva influencia del traslado, cambio y adaptación a un nuevo sistema social y escolar. Podemos decir, en términos generales, que los jóvenes reagrupados deben hacer frente a un conjunto de obstáculos adicionales si los comparamos con las trayectorias "aparentemente sin grandes saltos" de los jóvenes de segunda generación. Es sin duda el cambio geográfico y de escenario social y educativo un factor determinante en la duración y, en ocasiones, en el abandono de la trayectoria educativa. Desde el prisma del análisis de género, hemos ya comentado la relación entre la esfera de lo educativo y el ámbito privado, que en cierta forma vuelve la continuidad de estudios tareas compatibles con las obligaciones que según los géneros son establecidas para los descendientes.

Ahora bien, si bien el cambio de sistema educativo ocasiona desafíos importantes para los jóvenes marroquíes en Galicia, esta variable no debe considerarse la única y principal, pues, a partir 
de las historias narradas, comprobamos que las consecuencias de una inserción segmentada de los padres impactan de lleno en las posibilidades de continuación de los estudios después de la etapa de educación obligatoria. Es por ello que nuestras conclusiones apuntan a confirmar la hipótesis ya establecida por los autores ya mencionados a lo largo de nuestro análisis (Pedreño Cánovas, 2013; García Borrego, 2012) acerca de una real reproducción de las desigualdades sociales de generación en generación y de su relación con una estructura laboral étnicamente estratificada (Villares, 2010).

Para comprobarlo, nos centraremos en la medición de la movilidad social intergeneracional desde una perspectiva educativa y profesional. Dichos resultados han mostrado que las migraciones marroquíes describen una minoría de casos de movilidad tímidamente ascendente respecto de la irregularidad laboral de los padres, acompañada de una preocupante reproducción de esta situación de irregularidad laboral (cinco de diez casos) en los hijos. Se trata de jóvenes que en su mayoría se sitúan dentro de la economía sumergida tras la finalización de la trayectoria educativa. Así, comprobamos que este proceso se produce como consecuencia de varios factores, entre ellos: i) la situación socio-económica familiar que lleva a los descendientes a insertarse en el mercado laboral en una instancia prematura; ii) el abandono de la trayectoria formativa en un momento inmediato tras la educación secundaria obligatoria, vinculado a lo anterior; iii) la existencia de unos canales de acceso al mercado de trabajo etno-estratificados (redes sociales de tipo étnicas); iv) la discriminación dentro del mercado de trabajo y por último, v) la situación estructural de desempleo juvenil en Galicia como contexto de especial precariedad laboral. Con esto podemos decir que la reproducción de las desigualdades se plantea como un elemento evidente en las conclusiones extraídas, pese a que cierta agencia se puede evidenciar en el caso de las niñas, cuyos niveles educativos han superado en la mayoría de los casos, el de sus padres y que podrían mostrar una mayor potencialidad hacia la promoción social. Pese a ello, en el marco de una economía local deteriorada por la crisis mundial a momentos del estudio, el nivel de estudios alcanzado no logra traspasar las barreras de discriminación y segmentación dentro del mercado laboral.

Nuestra hipótesis de partida se confirma en que el proceso de reproducción de las desigualdades se ve entonces ilustrado en unas trayectorias educativas truncadas por las dificultades encontradas 
en el proceso de adaptación al nuevo sistema escolar pero sobre todo por las consecuencias que acarrea una integración segmentada de estas familias.

Ahora bien, cabe destacar la complejidad del caso marroquí en cuanto a dos situaciones particulares: la primera, que tiene que ver con el análisis de la movilidad social ocupacional de las mujeres, teniendo como referente unas madres inactivas (según los criterios de una estructura laboral europea y occidental). Ello supone que la entrada al mercado de trabajo de las hijas conlleva una mejora inevitable de las condiciones en el ámbito de la actividad laboral, si bien éstas se ven aplastadas por los procesos de discriminación y segregación social según origen étnico, además de por el hecho de ser mujeres.

La segunda de las dificultades añadidas a este análisis hace referencia a la ubicación de los descendientes en puestos de trabajo sin cualificación, si bien superan la posición de los padres dentro del mercado laboral, ubicados en actividades informales dentro de la economía secundaria. Este enfoque se alinea con las teorías de Piore y otros autores (Piore, 1979) que analizan la posición y paso desde el mercado secundario al primario. La movilidad ocupacional se produce con el acceso a un contrato de trabajo y en el paso desde el mercado informal al mercado formal, lo que supone una mejora en las condiciones de empleo.

Hemos podido comprobar que los únicos casos reconocidos en este estudio como de "movilidad ascendente" corresponden a aquellos que describen el paso desde la economía secundaria a la primaria, sin que ello suponga una verdadera mejora en la ubicación dentro de la estructura económica en términos de ingresos. Así, la conclusión más evidente que resulta de este estudio es que los y las jóvenes son empleados y empleadas en puestos que ocupan los peldaños más bajos de la escala social gallega.

A modo de cierre de este análisis, podemos confirmar que las condiciones estructurales parecen incidir con fuerza sobre las trayectorias ocupacionales del colectivo marroquí en Galicia. Este hecho podría guardar relación también con el origen social de las familias (proletariado agrícola) y con el tipo y calidad de los capitales activados tras la migración. Más aun, estas conclusiones nos permiten observar que parte de la movilidad social producida por los hijos está influida por la capacidad de las familias de escapar al destino de una estructura laboral jerarquizada étnicamente, observando cierta esperanza de agencia social en la jóvenes mujeres. 


\section{REFERENCIAS}

Aparicio, R. y Portes, A. (2014). Crecer en España: La integración de los hijos de inmigrantes. Barcelona: Obra Social” La Caixa”.

Berriane, M. (2004). "La larga historia de las migraciones marroquíes", en López García, B. y Berriane, M. (Dirs.) Atlas de la inmigración marroquí en España, (pp. 24-25). Madrid: Universidad Autónoma de Madrid.

Bayona-i-Carrasco, J., Domingo, A. y Menacho, T. (2020). Trayectorias migratorias y fracaso escolar de los alumnos inmigrados y descendientes de migrantes en Cataluña. Revista Internacional de Sociología, 78(1), 150.

Boukhobza, N. (2005). Les filles naissent après les garçons. Représentations sociales des populations d'origine maghrébine en France. Revue européenne des migrations internationales, 21(1), 227-242.

Bourdieu, P. y Passeron, J. C. (1970). La Reproduction: Éléments d'une théorie du système d'enseignement. Paris: Les Editions de minuit.

Bourdieu, P., Passeron, J. C., Melendres, J. y Subirats, M. (1981). La reproducción: elementos para una teoría del sistema de enseñanza. Barcelona: Laia

Echeverría Zabalza, J. (1998). Movilidad social y comportamiento electoral, Reis, 237-267.

Echeverría Zabalza, J. (1999). La movilidad social en España, 1940-1991, (163). Madrid: Ediciones AKAL.

Empez Vidal, N. (2015). “Solo valiente!” Los menores que migran solos de Marruecos a Cataluña. Tesis doctoral. Barcelona: Universitat Autònoma de Barcelona.

Fernández Enguita, M. (2001). La educación intercultural en la sociedad multicultural, Organización y gestión educativa. Revista del Fórum Europeo de Administradores de la Educación, 9(6), 3-7.

García Borrego, I. (2011). La difícil reproducción de las familias inmigrantes. ¿Hacia la formación de un proletariado étnico español? Papers. Revista de sociología, 96(1), 55-76.

García Borrego, I. (2012). Las estrategias familiares de reproducción de marroquíes y ecuatorianos en Murcia. En García, A. A., Gadea, M. E. y Pedreño, A. (Eds.) (2010), Tránsitos migratorios: contextos transnacionales y proyectos familiares en las migraciones actuales (pp. 171-203). Murcia: Editum.

García Castaño, F. J. y Rubio Gómez, M. (2013). Juntos pero no revueltos: procesos de concentración escolar del" alumnado extranjero" en determinados centros educativos. Revista Dialectología y Tradiciones Populares, 68(1), 7-31 (2013). [http://hdl.handle.net/10481/34477]

Gil Araujo, S. (2007). Las argucias de la integración. Construcción nacional y gobierno de lo social a través de las políticas e integración de inmigrantes. Los casos de Cataluña y Madrid. Tesis doctoral, Madrid: Universidad Complutense de Madrid. 
Gil-Hernández, C. J. y Gracia, P. (2018). “Adolescents' educational aspirations and ethnic background: The case of students of African and Latin American migrant origins in Spain." Demographic Research 38(23), 577 618. https://doi.org/10.4054/DemRes.2018.38.23

González-Ferrer, A. y Cebolla-Boado, H. (2018). "Los hijos de la inmigración en España: valores, aspiraciones y resultados”. Pp. 111-163 en Informe España 2018, coordinado por A. Blanco et al. Madrid: Universidad Pontificia Comillas.

López García, B. (2004). La evolución de la inmigración marroquí en España. 1991-2003. En García y Berriane (2004), Atlas de inmigración Marroquí en España, Barcelona: Ed. Taller de Estudios internacionales Mediterráneos.

Mateo Dieste, J. L. (2018). Moros vienen. Historia y política de un estereotipo. Melilla: Instituto de las culturas.

Meurs, D., Pailhé, A. et Simon, P. (2005). Mobilité intergénérationnelle et persistance des inégalités. Documents de travail 130, 36 p.: www.ined.fr

Miguel, V. de y M. Solana. (2017). Immigrants in the Educational System in Spain: Who Persists? Social Indicators Research 132, 733-755. https://doi. org/10.1007/s11205-016-1323-4

Miguélez, F. (2011). Trayectorias laborales de los inmigrantes en España. Quit, Centre d'Estudis Sociòlogics sobre la Vida Quotidiana i el Treball. Barcelona: Universitat Autònoma de Barcelona.

Oso, L., Dalle, P. y Boniolo, P. (2018). Movilidad social de familias gallegas en Buenos Aires pertenecientes a la última corriente migratoria: estrategias y trayectorias. Papers. Revista de Sociologia 104(2), 305-335.

Pedreño Cánovas, A. (2007). Jóvenes españoles e inmigrantes en el espacio público: una investigación sobre la realidad murciana. En López Sala, A. y Cachón Rodríguez, L. Juventud e Inmigración: Desafíos para la participación y para la Integración (pp. 137-157). La Laguna: Dirección General de Juventud de la Consejería de Empleo y Asuntos Sociales del Gobierno de Canarias.

Pedreño Cánovas, A. (2010). Familias inmigrantes: el trabajo de los padres y las estrategias de trabajo de los hijos en las áreas mediterráneas de agricultura intensiva. En Lara Flores, S.M. (Coord.), Migraciones de Trabajo y movilidad territorial, (pp. 333-367). Consejo Nacional de Ciencia y Tecnología. Méjico: Ed. M. A.Porrua.

Pedreño Cánovas, A. (coord.) (2013). “Que no sean como nosotros". Trayectorias formativo-laborales de los hijos de familias inmigrantes en el campo murciano. Murcia: EDITUM publicaciones de la Universidad de Murcia.

Piore, M. J. (1979). Birds of passage: migrant labour and industrial societies. Cambridge: Cambridge University Press.

Portes, A. y Böröcz, J. (1989). Contemporary immigration: Theoretical perspectives on its determinants and modes of incorporation. International migration review, 23(3), 606-630.

Portes, A., Castells, M., \& Benton, L. A. (Eds.). (1989). The informal economy: Studies in advanced and less developed countries. JHU Press. 
Portes, A., Aparicio, R. y Haller, W. (2009). La segunda generación en Madrid: un estudio longitudinal. Boletín Elcano, Área: Demografía, Población y Migraciones Internacionales ARI 67/2009; (113), 10.

Pumares, P. (2002). La inmigración marroquí en la Comunidad de Madrid. Universidad Complutense de Madrid, Servicio de Publicaciones.

Pumares, P., García Coll, C. y Hita, A. (2006). La movilidad laboral y geográfica de la población extranjera en España. Ministerio de Trabajo y Asuntos Sociales.

Pumares, P., Marín Cassinello, E. y Rojas Tejada, A. J. (2011). El proyecto migratorio a corto plazo de la población marroquí en Almería: factores explicativos. En F. J. García Castaño y N. Kressova. (Coords.). Actas del I Congreso Internacional sobre Migraciones en Andalucía (pp. 575-587). Granada: Instituto de Migraciones. SBN: 978-84-921390-3-3.

Rodríguez-Reche, C. y Rodríguez-García, D. (2020). El estigma de la musulmaneidad: visibilidad percibida y racismo en hijas de parejas mixtas con padre/madre de origen magrebí en Barcelona y Granada. Perifèria, revista de recerca $i$ formació en antropologia, 25(1), 4-27. https://doi. org/10.5565/rev/periferia.723

Rumbaut, R. (2004). Ages, Life Stages, and Generational Cohorts: Decomposing the Immigrant First and Second Generations in the United States. International Migration Review, 38(3), 1160-1205.

Sáiz López, A. (2004). La migración china en España: Características generales. Revista CIDOB d'afers internacionals, (4)151-163.

Sánchez Hugalde, A. (2004). Movilidad intergeneracional de ingresos y educativa en España (1980-90). En XI Encuentro de Economía Pública: los retos de la descentralización fiscal ante la globalización, Barcelona: Encuentro de Economía Pública.

Valenzuela, J. R. A. (1999). Gender Roles and Settlement Activities Among Children and Their Immigrant Families. American Behavioral Scientist (42), 720.

Villares Varela, M. (2010). Inmigración y empresa en Galicia: la movilización diferencial del capital financiero, humano y social. Tesis doctoral. A Coruña: Universidade da Coruña. 
